\title{
Aparência tomográfica dos discos intervertebrais mineralizados em cães da raça Dachshund Miniatura
}

\author{
[Tomographic appearance of calcified intervertebral discs in miniature dachshund] \\ D. Baraúna Júnior ${ }^{1}$, T.H.T. Fernandes ${ }^{2}$, I.C.C. Silva ${ }^{2}$, M.A. Bonelli ${ }^{2}$, C.R.O. Santos ${ }^{1}$, \\ M.M.A. Amorim ${ }^{2}$, F.S. Costa ${ }^{4}$, E.A. Tudury ${ }^{4}$ \\ ${ }^{1}$ Universidade Federal do Vale do São Francisco - Univasf - Petrolina, PE \\ ${ }^{2}$ Aluno de pós-graduação - Universidade Federal do Vale do São Francisco - Univasf - Petrolina, PE \\ ${ }^{3}$ Universidade Federal Rural de Pernambuco - UFRPE - Recife, PE
}

\begin{abstract}
RESUMO
Objetivou-se com este trabalho descrever os aspectos de tomografia computadorizada de discos intervertebrais de cães da raça Dachshund com um ano de idade, assim como quantificar, qualificar e localizar a mineralização nos componentes do DIV e no espaço intervertebral da coluna vertebral. Dez cães dessa raça, com um ano de idade, foram submetidos à tomografia computadorizada (TC). Nas imagens de reconstrução sagital dos animais, procedeu-se à identificação de sete DIV em cada animal, entre as vértebras $\mathrm{T}_{9}$ e $\mathrm{L}_{3}$, totalizando a análise de 70 DIV. Entre os DIV mineralizados, a presença da alteração foi determinada quanto à localização no núcleo pulposo (NP), no anel fibroso (AF) e em AF/NP. Dos 70 DIV avaliados, 45 foram considerados como mineralizados. Entre os classificados como mineralizados, $20 \%(9 / 45), 17,8 \%(8 / 45)$ e $62,2 \%(28 / 45)$ estavam localizados nas regiões do NP, AF e $\mathrm{AF} / \mathrm{NP}$, respectivamente. A mineralização encontrada neste estudo esteve caracterizada por aumento de atenuação radiográfica com densidade mineral na topografia do DIV, havendo variações da localização e da radiodensidade de área calcificada entre animais e DIV no mesmo indivíduo. As alterações relativas à mineralização dos DIV podem ser classificadas tomograficamente quanto à localização da alteração no disco em AF, NP e AF/NP.
\end{abstract}

Palavras-chave: cão, coluna vertebral, diagnóstico por imagem, radiografia

\begin{abstract}
The objective was to describe CT imaging of calcified IVD in one-year-old Dachshunds, as well as describe the location in the soft tissue structures that make up the IVD. Ten one-year-old dogs underwent computed tomography (CT). In sagittal reconstruction images of animals, the identification of 70 IVD present in the spaces between L3 - T9 were performed. Among calcified IVDs, the presence of the change was determined as the location in the nucleus pulposus (NP), annulus fibrosus (AF) and both. Of a total of 70 evaluated IVDs, 45 were found to calcified and 25 not calcified. Among calcified discs, location was determined in nine (20\%) in NP, eight discs (17.77\%) in AF and $28(62,23 \%)$ NP \& AF. Calcification in this study was characterized by increased radiographic attenuation mineral density in the IVD topography. There is variation in the location and radiodensity of calcified areas between animals and IVD in the same individual. Changes related to IVD calcification can be classified as tomographic location in $A F, N P$ and NP \& $A F$.
\end{abstract}

Keywords: dog, spine, imaging diagnosis, radiography

Recebido em 14 de outubro de 2016

Aceito em 19 de outubro de 2016

E-mail: durvalbarauna@hotmail.com. 


\section{INTRODUÇÃO}

O disco intervertebral (DIV) saudável é composto por um núcleo pulposo (NP) macio e gelatinoso, cercado por um anel fibroso (AF) fibrocartilaginoso, formado por camadas lamelares bem organizadas, além das placas cartilaginosas terminais (PCT), que formam as fronteiras craniais e caudais do disco (McKee, 2000; Bergknut et al., 2015). A zona de transição (ZT) é uma região interposta entre o AF e o NP (Innes e Melrose, 2015).

A degeneração do disco intervertebral é um processo comum que ocorre com a idade. Mudanças degenerativas no disco intervertebral de cães condrodistróficos e não condrodistróficos são geralmente referidos como metaplasia condroide e metaplasia fibroide, respectivamente (Brisson, 2010).

Essa degeneração é caracterizada por alterações da composição bioquímica e na integridade mecânica do DIV. No disco degenerado, o teor de glicosaminoglicano diminui e aumenta a quantidade de colágeno desnaturado (Bach et al., 2014) com a subsequente calcificação distrófica do núcleo (Hansen, 1952). A desidratação leva a lacerações do anel fibroso e/ou extrusões do disco (Bach et al., 2014). Material mineralizado no DIV não é um sinal de hérnia de disco. Nem todos os DIV mineralizados vão extrudir e nem todo o material de DIV extrudido é mineralizado (Widmer e Thrall, 2002).

Com um ano de idade, aproximadamente 90\% dos cães condrodistróficos têm mudanças que podem afetar múltiplos discos. Mineralização parcial ou completa do disco é comum (McKee, 2000). Eventualmente, pequenos focos de degeneração e fibrose ocorrem, o que faz o disco aparecer opaco em vez de gelatinoso e pode obscurecer a fronteira entre o NP e o AF (Evans, 1993). Alterações também ocorrem no AF com perda de fibras e fragmentação de sua estrutura (McKee, 2000).

Desde que os tipos de degeneração do DIV foram descritos, por Hansen, em 1952, tornou-se evidente para os médicos veterinários que nem todas as degenerações do disco se encaixavam perfeitamente nessas duas categorias. Por exemplo, protrusões de DIV muitas vezes parecem conter material nuclear mineralizado, e extrusão aguda do núcleo que foi submetido à degeneração fibrosa pode ocorrer, como também hérnias agudas de fragmentos do AF. Ruptura do seio venoso que se sobrepõe ao DIV, quando ocorre extrusão, pode resultar na formação de um hematoma. Lesões do DIV podem causar um amplo espectro de alterações (Olby e Jeffery, 2012).

O disco intervertebral pode ser facilmente avaliado nas imagens por tomografia computadorizada (TC), e a mineralização do NP é facilmente identificada como material hiperdenso (Olby et al., 2000). A característica tomográfica da extrusão aguda do disco intervertebral inclui material hiperdenso dentro do canal vertebral, perda da gordura epidural e distorção da medula espinal (Dewey et al., 2016).

A TC apresenta uma sensibilidade maior e pode detectar alterações que não seriam visualizadas no exame radiográfico convencional, uma vez que permite captar diferenças de densidade dos tecidos de $0,05 \%$ enquanto a radiografia capta diferenças de 0,5\% (Hathcock e Stickle, 1993).

Objetivou-se com este trabalho descrever o aspecto tomográfico de discos intervertebrais de cães da raça Dachshund com um ano de idade, assim como a localização da mineralização nos componentes do DIV e no espaço intervertebral da coluna vertebral.

\section{MATERIAL E MÉTODOS}

Esta pesquisa foi aprovada pelo Comitê de Ética para Uso de Animais (Ceua) da Universidade Federal Rural de Pernambuco, com o $\mathrm{n}^{\circ}$ 001/2015 (Anexo B). Vale ressaltar que, ao final da pesquisa, os 10 animais foram doados para tutores.

Dez cães da raça Dachshund Miniatura, com quatro meses de idade, adquiridos em diferentes provedores, foram mantidos durante oito meses em canis com acesso à luz natural, recebendo água e alimento completo para cães. Com um ano de idade, esses animais foram submetidos à tomografia computadorizada (TC) da coluna vertebral no segmento toracolombar.

Para realização dos exames de TC, os cães foram submetidos a jejum alimentar de 12 horas e 
hídrico de duas horas. Estabeleceu-se acesso venoso e fluidoterapia com solução fisiológica $10 \mathrm{~mL} / \mathrm{kg}{ }^{1 /} \mathrm{h}^{1}$, indução com propofol, via intravenosa, na dose de $4 \mathrm{mg} / \mathrm{kg}^{1}$ de peso, e os animais foram mantidos com anestesia inalatória com isoflurano diluído em oxigênio a 100\%.

As imagens coletadas foram obtidas com os animais posicionados em decúbito ventral. Utilizou-se equipamento de TC helicoidal (GE Hi-Speed FXI - General Eletrics, 06828-0001, Fairfield, Connecticut - single slice), com fator de exposição de radiação predeterminado em $120 \mathrm{~mA}$ e $120 \mathrm{kVp}$, com filtro leve e pitch de $1.0 \mathrm{~mm}$. Inicialmente foi feito o scout para visualização do correto posicionamento e determinação do tamanho da área da coluna vertebral (torácica nove a lombar três) a ser avaliada, ou seja, o campo de visão, para então dar início ao escanograma com cortes transversais de $1 \mathrm{~mm}$ de espessura por $1 \mathrm{~mm}$ de intervalo (1x1). As imagens foram reconstruídas com cortes sagitais e transversais em janela óssea (WW 3500, WL 800). As imagens tomográficas foram avaliadas com auxílio de programa de diagnóstico por imagem digital para tratamento de imagens médicas (Osirix MD - Osirix Imaging Software - Genebra, Suíça). Antecedendo os exames, o aparelho foi devidamente calibrado para melhor padronização dos dados.
Nas imagens de reconstrução sagital dos animais, procedeu-se à identificação dos discos intervertebrais presentes nos espaços entre as vértebras $\mathrm{T}_{9}$ e $\mathrm{L}_{3}$, totalizando 70 discos, que foram avaliados e classificados quanto à aparência tomográfica em normais e mineralizados. Entre os discos alterados, a presença da mineralização foi determinada quanto à região no núcleo pulposo $(\mathrm{NP})$, no anel fibroso (AF) e em ambos (NP/AF), assim como quantificou-se o número de discos com tal alteração e sua localização no espaço intervertebral. As imagens tomográficas também foram avaliadas, de acordo com a classificação radiográfica proposta por Stigen e Kolbjornsen (2007), em graus leve, moderado e grave.

\section{RESULTADOS}

Dos 70 discos avaliados, 64,3\% (45/70) foram considerados como mineralizados, com $100 \%$ dos animais apresentando mineralização em algum grau. Entre os classificados como mineralizados, a localização nos componentes do DIV foi determinada em nove discos $(20 \%)$ no $\mathrm{NP}$, em oito discos $(17,8 \%)$ no $\mathrm{AF}$ e em 28 discos $(62,2 \%)$ no NP/AF. Já a localização da mineralização no espaço intervertebral foi maior entre os espaços $\mathrm{T}_{09^{-}}{ }_{13}$, determinada em nove entre $\mathrm{T}_{09^{-} 10}$, oito entre $\mathrm{T}_{10^{-} 11}$, seis entre $\mathrm{T}_{11^{-12}}$, $\mathrm{T}_{12^{-13}}$ e $\mathrm{L}_{2^{-} 3}$ e cinco entre $\mathrm{T}_{13^{-}-\mathrm{L}_{1}}$ e $\mathrm{L}_{1^{-} 2}$, conforme apresentado no Quadro 1.

Quadro 1. Distribuição das calcificações de acordo com a localização no espaço intervertebral e nos componentes do disco intervertebral

\begin{tabular}{|c|c|c|c|c|c|c|c|}
\hline \multirow{2}{*}{ ANIMAL } & \multicolumn{7}{|c|}{ DISCO MINERALIZADO } \\
\cline { 2 - 8 } & $\mathbf{T}_{9-10}$ & $\mathbf{T}_{\mathbf{1 0 - 1 1}}$ & $\mathbf{T}_{\mathbf{1 1 - 1 2}}$ & $\begin{array}{c}\mathbf{T}_{\mathbf{1 2}-} \\
\mathbf{1 3}\end{array}$ & $\mathbf{T}_{\mathbf{1 3}}-\mathbf{L}_{\mathbf{1}}$ & $\mathbf{L}_{\mathbf{1 - 2}}$ & $\mathbf{L}_{\mathbf{2 - 3}}$ \\
\hline $\mathbf{1}$ & $\mathrm{NP} / \mathrm{AF}$ & - & $\mathrm{NP} / \mathrm{AF}$ & - & - & - & - \\
\hline $\mathbf{2}$ & $\mathrm{NP} / \mathrm{AF}$ & $\mathrm{NP} / \mathrm{AF}$ & $\mathrm{NP} / \mathrm{AF}$ & $\mathrm{NP} / \mathrm{AF}$ & $\mathrm{NP} / \mathrm{AF}$ & $\mathrm{AF}$ & $\mathrm{NP} / \mathrm{AF}$ \\
\hline $\mathbf{3}$ & $\mathrm{NP} / \mathrm{AF}$ & $\mathrm{NP} / \mathrm{AF}$ & - & $\mathrm{NP}$ & - & $\mathrm{NP} / \mathrm{AF}$ & $\mathrm{NP} / \mathrm{AF}$ \\
\hline $\mathbf{4}$ & $\mathrm{AF}$ & - & - & - & - & - & - \\
\hline $\mathbf{5}$ & $\mathrm{NP} / \mathrm{AF}$ & $\mathrm{NP} / \mathrm{AF}$ & - & - & $\mathrm{AF}$ & - & $\mathrm{NP} / \mathrm{AF}$ \\
\hline $\mathbf{6}$ & $\mathrm{NP}$ & $\mathrm{AF}$ & $\mathrm{AF}$ & $\mathrm{NP}$ & $\mathrm{NP}$ & - & $\mathrm{NP}$ \\
\hline $\mathbf{7}$ & $\mathrm{NP} / \mathrm{AF}$ & $\mathrm{NP} / \mathrm{AF}$ & $\mathrm{NP}$ & $\mathrm{AF}$ & $\mathrm{NP} / \mathrm{AF}$ & $\mathrm{NP}$ & - \\
\hline $\mathbf{8}$ & - & $\mathrm{NP} / \mathrm{AF}$ & $\mathrm{NP} / \mathrm{AF}$ & - & $\mathrm{NP} / \mathrm{AF}$ & $\mathrm{NP} / \mathrm{AF}$ & $\mathrm{NP} / \mathrm{AF}$ \\
\hline $\mathbf{9}$ & $\mathrm{NP} / \mathrm{AF}$ & $\mathrm{NP} / \mathrm{AF}$ & $\mathrm{NP} / \mathrm{AF}$ & $\mathrm{AF}$ & - & $\mathrm{NP} / \mathrm{AF}$ & - \\
\hline $\mathbf{1 0}$ & $\mathrm{NP} / \mathrm{AF}$ & $\mathrm{AF}$ & - & $\mathrm{NP}$ & - & - & $\mathrm{NP}$ \\
\hline
\end{tabular}

Anel fibroso (AF), núcleo pulposo (NP) e (NP/AF). 
A mineralização encontrada no presente estudo esteve caracterizada por aumento de atenuação radiográfica com densidade mineral na topografia do disco, havendo variações da localização e radiodensidade de área calcificada entre animais e discos intervertebrais no mesmo indivíduo. Nas imagens tomográficas em corte transversal ao nível do espaço intervertebral do segmento toracolombar da coluna de cães da raça
Dachshund Miniatura, foram demonstrados os diferentes padrões de mineralização de disco intervertebral, conforme ilustrado na Fig. 1.

Com base na classificação proposta por Stigen e Kolbjornsen (2007), a mineralização dos DIV foi classificada em leve e moderada, já que em nenhum DIV foi avaliada como mineralização distinta de todo o NP.

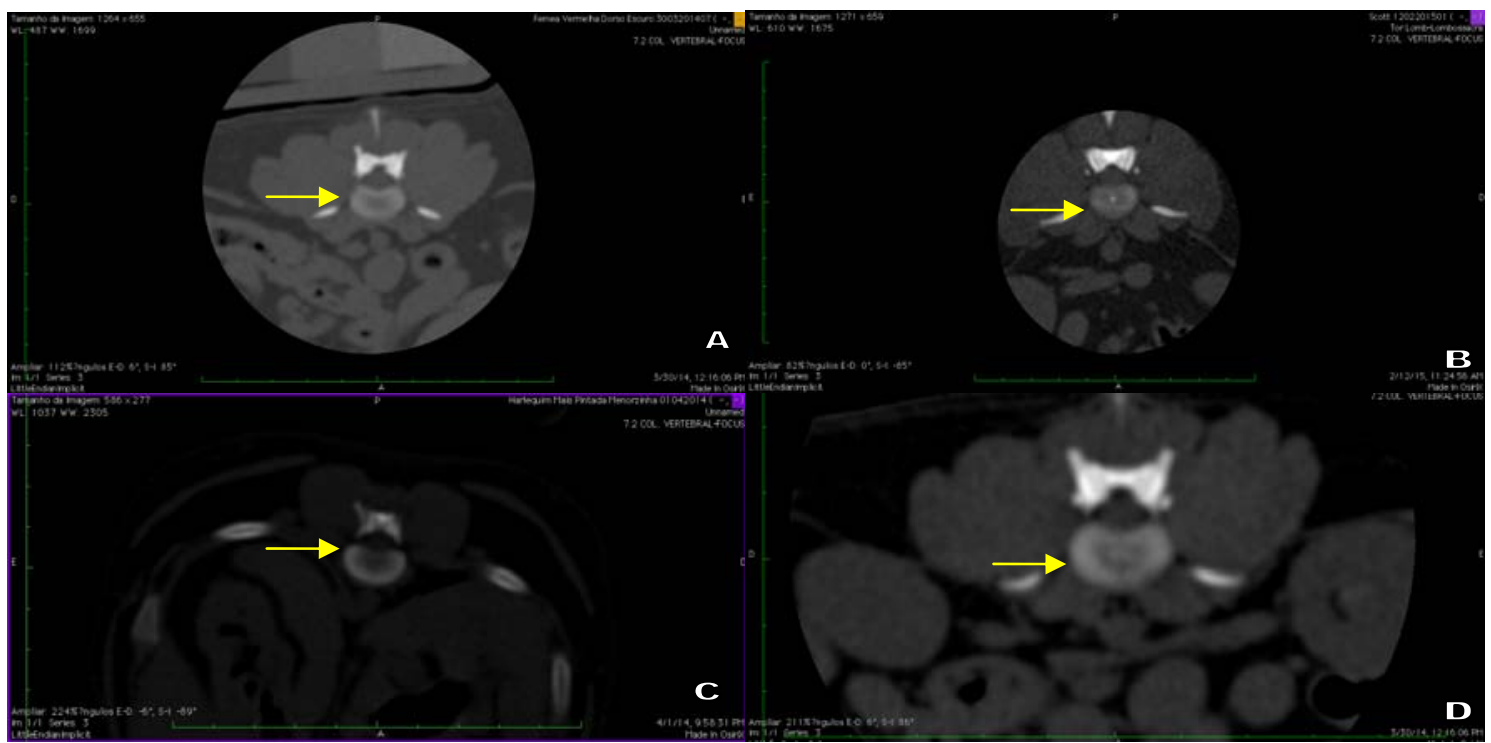

Figura 1. Imagens de tomografia computadorizada em corte transversal no nível do espaço intervertebral do segmento toracolombar da coluna vertebral de cães da raça Dachshund Miniatura, demonstrando os padrões observados de calcificação do disco: A. ausência de calcificação; B. calcificação da região central (núcleo pulposo - NP) - seta amarela; C. calcificação da região periférica (anel fibroso - AF) - seta amarela; D. calcificação das regiões central e periférica (NP/AF) - seta amarela.

\section{DISCUSSÃO}

A raça Dachshund foi escolhida para este estudo por apresentar de 10 a 12,6 vezes mais risco que outras raças, como relata Braund (1996). Além disso, a variedade Miniatura foi escolhida, pois, segundo Smolders et al. (2013), é mais frequentemente afetada pela extrusão de DIV do que os Standard, informação essa que foi corroborada, acrescentando-se ainda que a raça Dachshund é um excelente modelo para o estudo ora apresentado, devido ao grande número de discos mineralizados.

$\mathrm{O}$ número de discos mineralizados em Dachshund é um bom preditor de doença do disco intervertebral (DDIV) (Jensen et al., 2008). A herniação raramente ocorre em cães sem mineralização de disco, enquanto os animais com várias calcificações têm alto risco (Stigen, 1996), logo, com base nos achados deste estudo, estes cães têm alto risco em desenvolver a DDIV.

O risco de eutanásia devido à DDIV foi significantemente maior em Dachshund, em que foram encontrados mais de quatro discos mineralizados nas radiografias realizadas aos dois e quatro anos de idade (Jensen et al., 2008). Esse fato é preocupante, já que dos 10 animais utilizados neste experimento, seis apresentavam mais de quatro discos mineralizados, considerando que, no presente estudo, só se utilizaram para avaliação os discos intervertebrais entre as vértebras torácica nove e lombar três, ao passo que Jensen e colaboradores (2008) consideraram todos os discos da coluna vertebral, podendo esses animais apresentarem ainda mais DIV mineralizados. 
O nível da janela estabelece o tom de cinza central, que deve ser selecionado sempre de acordo com o tecido de interesse. A amplitude da janela refere-se à variação de unidades Hounsfield (HU), representada pelos níveis de cinza. Isso permite uma distinção mais precisa de pequenas variações nos tons de cinza que compõem o tecido avaliado, o que facilita a interpretação da imagem tomográfica. Assim, quando um grande número de estruturas precisa ser diferenciado, deve-se optar por uma janela mais ampla (Costa et al., 2012). Essa característica da TC permite identificar estágios precoces de mineralização, como os observados neste trabalho, que não seriam visualizadas no exame radiográfico.

O exame histopatológico é superior ao exame radiográfico na identificação de discos intervertebrais mineralizados em Dachshund, sendo considerado método ouro (Stigen e Kolbjornsen, 2007). Contudo não foi possível realizá-lo no presente estudo, em virtude do delineamento experimental, que não incluía cirurgia nem eutanásia dos animais.

A TC é o método por imagem mais sensível para demonstrar a mineralização de discos, pois eles podem ser identificados numa fase inicial, e o pico do número de discos mineralizados pode ser obtido numa idade mais precoce (Jensen e Christensen, 2000). É considerada a melhor escolha como método de diagnóstico em cães com suspeita clínica de ruptura do disco tipo I (Newcomb et al., 2012). Diferentemente do exame histopatológico, a TC pode ser realizada de forma não invasiva em pacientes clínicos, por isso foi a opção de escolha neste estudo.

A TC apresenta vantagens como contraste superior em tecidos moles em comparação à radiografia convencional. Quando não contrastada, pode ser realizada só com sedação, o que não é possível com a ressonância magnética (RM). Com relação ao custo da TC, este é normalmente menor que a RM, além de a TC ser uma modalidade de imagem mais rápida que a RM. As avaliações de osso e de hemorragia aguda são visualizadas melhor na TC do que na RM. A RM fornece detalhes superiores em tecidos moles quando comparada à TC (Dewey et al., 2016). Neste estudo, a RM não foi uma opção, pois é um método de imagem não disponível para animais no estado da realização do estudo, havendo poucos equipamentos disponíveis no Brasil de uso específico para animais. Além disso, o tempo de anestesia necessário para a realização da $\mathrm{RM}$ é muito maior quando comparado ao da TC.

A mineralização do DIV encontrada neste estudo está de acordo com o descrito por Olby et al. (2000), os quais citam que a atenuação radiográfica aumenta à medida que o grau de mineralização também aumenta.

Discos saudáveis apresentam estruturas bem distinguíveis umas das outras, contudo, no início da degeneração, as fronteiras entre essas estruturas se tornam progressivamente mais difíceis de serem diferenciadas (Bergknut et al., 2015). No exame de imagem, a calcificação distrófica de um disco em degeneração começa geralmente no centro do núcleo pulposo e se estende perifericamente. No entanto, o anel pode ser submetido à mineralização separadamente (Widmer e Thrall, 2002), corroborando os achados deste estudo no que tange à localização da mineralização no AF somente, contudo a maior parte dos animais apresentou mineralização do AF/NP.

Em cães condrodistróficos, as mudanças no DIV ocorrem rapidamente e, em todos os discos intervertebrais de todas as regiões da coluna vertebral, quase simultaneamente. A distinção entre o NP e o AF torna-se gradualmente menos aparente e a diferenciação das estruturas é quase impossível (Bray e Burbidge, 1998). Isso ocorreu nesta investigação nos cães que apresentavam as regiões do AF e do NP simultaneamente mineralizadas

Embora seja menos comum, cães condrodistróficos podem apresentar metaplasia fibroide (Hansen tipo II - protusão anular) (Brisson, 2010). Apesar de esse tipo de alteração ser mais frequente em cães com mais de sete anos, uma fibrose extensiva do NP tem sido ocasionalmente isolada de cães muito mais jovens, estando sempre presente nesses animais uma desorganização focal das camadas lamelares do AF. Em alguns cães, as mudanças degenerativas no AF foram observadas antes mesmo de qualquer evidência de desorganização do NP (Bray e Burbidge, 1998), contudo essas fissuras em AF resultam em aumento do estresse sobre o NP (Bergknut et al., 2013). Essa seria 
uma das possibilidades para explicar a alteração somente em AF, nos cães desta pesquisa.

Outra possibilidade para a ocorrência da mineralização somente no AF seria o desaparecimento de mineralização do $\mathrm{NP}$, que pode ocorrer sem sinais de extrusão, possivelmente resultado da degeneração progressiva do disco, em vez de regeneração do disco (Brisson, 2010). No entanto, não se acredita que tenha acontecido dessa forma nos resultados deste trabalho em função da idade precoce, pois, em trabalho realizado por Jensen e Arnbjerg (2001), esse fenômeno de dissolução de discos previamente mineralizados não ocorreu em nenhum cão dos seis aos 12 meses de idade.

A maior ocorrência de mineralização nos NP/AF e nos NP pode ser explicada pela ocorrência da metaplasia condroide nas raças condrodistróficas, que tende a se iniciar na periferia e se espalhar por todo o NP, estando completa na maioria dos DIV por volta de um ano de idade (Smolders et al., 2013). Além disso, as forças anormais geradas pela degeneração e mineralização do NP causam rupturas no $\mathrm{AF}$, formando canais através dos quais o núcleo anormal pode extruir. Ocasionalmente, fragmentos desse material, que avançam através do AF externo e interno, são vistos no exame post mortem, mas são raramente retos e, em geral, difíceis de serem seguidos. A composição química desse material é idêntica à do material remanescente do NP, o que confirma a migração do NP através do AF (Brisson, 2010), que pode ter ocorrido nos animais desta pesquisa com mineralização do NP/AF.

Stigen e Kolbjornsen (2007) classificam em três graus de mineralização os discos intervertebrais avaliados por meio de radiografia convencional: leve, quando apresenta um ou mais corpos mineralizados com diâmetro menor que $2 \mathrm{~mm}$, ou mineralização de parte da zona de transição entre NP e AF ou mineralização indistinta de grande parte do NP; moderada, quando apresenta corpos mineralizados menores que $2 \mathrm{~mm}$ e mineralização de parte da zona de transição, ou mineralização distinta de grande parte do NP ou indistinta mineralização de todo o NP; e como grave, quando ocorre uma mineralização distinta de todo o NP. Com base na classificação desses autores, extrapolada para a TC, os resultados deste trabalho se enquadram em mineralização leve e moderada, não havendo nenhuma em grau grave, uma vez que não houve mineralização distinta de todo o NP, nos DIV avaliados.

A calcificação distrófica ocorre em células mortas ou que estão morrendo, sendo importante como um indicador de lesão tecidual prévia. Os depósitos de cálcio são relativamente permanentes, mas inofensivos, a menos que interfiram mecanicamente no local da lesão (Myers e McGavin, 2009), logo, independentemente do grau de classificação da mineralização encontrado neste trabalho, a presença desta indica a ocorrência de uma lesão prévia, e, nesse caso, não deve ser considerada inofensiva, uma vez que ela pode interferir diretamente na biomecânica normal dos discos intervertebrais, trazendo graves consequências.

\section{CONCLUSÃO}

Com base nos resultados obtidos, todos os animais da raça Dachshund Miniatura apresentaram mineralização do DIV caracterizadas por aumento de atenuação radiográfica com densidade mineral na topografia do disco, havendo variações da localização e da radiodensidade de área calcificada entre animais e discos intervertebrais no mesmo indivíduo, sendo mais frequentes entre as vértebras $T_{9}-T_{13}$. As alterações relativas à mineralização dos discos intervertebrais podem ser classificadas tomograficamente quanto à localização da alteração no disco em AF, NP e $\mathrm{NP} / \mathrm{AF}$, sendo possível ainda utilizar a TC para classificar a mineralização em graus leve, moderado e grave. Tendo em vista uma maior sensibilidade dessa técnica, propõe-se que ela possa ser utilizada como ferramenta para seleção de exemplares que serão utilizados em programas de criação.

\section{REFERÊNCIAS}

BACH, F.C.; WILLEMS, N.; PENNING, L.C. et al. Potential regenerative treatment strategies for intervertebral disc degeneration in dogs. Vet. Res. Commun., v.10, p.1-12, 2014.

BERGKNUT, N.; FORTERRE, F.; LEVINE, J.M. et al. Comparasions between biped (human) and quadruped (canie/feline) intervertebral disc disease. In: FINGEROTH, J.; THOMAS, W. (Eds.). Advances in intervertebral disc disease in dogs and cats. Iowa: Wiley Blackwell, 2015. p.14-22. 
BERGKNUT, N.; SMOLDERS, L.A.; GRINWIS, G.C.M. et al. Intervertebral disc degeneration in the dog. Part 1: Anatomy and physiology of the intervertebral disc and characteristics of intervertebral disc degeneration. Vet. J., v.195, p.282-291, 2013.

BRAUND, K.G. Molestia do disco intervertebral. In: BOJRAB, M.J. Mecanismos da moléstia na cirurgia de pequenos animais. 2.ed. São Paulo: Roca, 1996. p.1104-1116.

BRAY, J.P.; BURBIDGE, H.M. The canine intervertebral disk. Part Two: Degenerative changes-nonchondrodystrophoid versus chondrodystrophoid disks. J. Am. Anim. Hosp. Assoc,. v.34, p.135-144, 1998.

BRISSON, B.A. Intervertebral disc disease in dogs. Vet. Clin. N. Am. Small, v.40, p.829-858, 2010.

COSTA, L.A.V.S.; OLIVEIRA, D.C.; CARDOSO, M.J.L.; COSTA, F.S. Densitometria óssea pelo método de tomografia computadorizada quantitativa em cães e gatos: revisão. Clín. Vet., v.17, p.82-86, 2012.

DEWEY, C.W.; COSTA, R.C.; DUCOTÉ, J.M. Neurodiagnostics. In: DEWEY, C.W.; COSTA, R.C. Practical guide to canine and feline neurology. 3.ed. Dely: Wiley Blackwell, 2016. p.61-86.

EVANS, H.E. Arthrology. In: Evans, H.E. Miller's anatomy of the dog. Philadelphia: Saunders, 1993. p.219-257.

HANSEN H.J. A pathologic-anatomical study on disc degeneration in dog, with special reference to the socalled enchondrosis intervertebralis. Acta Orthop. Scand. Suppl., v.11, p.1-117, 1952.

HATHCOCK, J.T.; STICKLE, R.L. Principles and concepts of computed tomography. Vet. Clin. N. Am. Small, v.23, p.399-415, 1993.

INNES, J.F.; MELROSE, J. Embryology, innervation, morphology, structure, and function of the canine intervertebral disc. In: FINGEROTH, J.; THOMAS, $\mathrm{W}$. Advances in intervertebral disc disease in dogs and cats. Iowa: Wiley Blackwell, 2015. p.3-7.

JENSEN, V.; AMBJERG, J. Development of intervertebral disk calcification in the Dachshund: a prospective longitudinal radiographic study. J. Am. Anim. Hosp. Assoc., v.37, p.274-282, 2001.

JENSEN, V.; BECK, S.; CHRISTENSEN, K.; AMBJERG, J. Quantification of the association between intervertebral disc calcification and disc herniation in Dachshund. J. Am. Vet. Med. Assoc., v.233, p.1090-1095, 2008.
JENSEN, V.F.; CHRISTENSEN, K.A. Inheritance of disc calcification in the Dachshund. J. Vet. Med. A, v.47, p.331-340, 2000.

McKEE, M. Intervertebral disc disease in the dog. 1. Pathophysiology and diagnosis. In Practice. v.22, p.355-369, 2000.

MYERS, R.K.; McGAVIN, M.D. Respostas celulares e teciduais. In: McGAVIN, M.D.; ZACHARY, J.F. Bases da patologia em veterinária. 4ed. São Paulo: Elsevier, 2009. p.3-62.

NEWCOMB, B.; ARBLE, J.; ROCHAT, M. et al. Comparasion of computed tomography and myelography to a reference standard of computed tomographic myelography for evaluation of dogs with intervertebral disc disease. Vet. Surg., v.41, p.207-214, 2012.

OLBY, N.J.; JEFFERY, N.D. Pathogenesis and physiology of central nervous system disease and injury. In: TOBIAS, K.M.; JOHNSTON, S.A. Veterinary surgery small animal. 7ed. St. Louis: Elsevier Saunders, 2012. p.374-387.

OLBY, N.J.; MUNANA, K.R.; SHARP, N.J.; THRALL, D.E. The computed tomographic appearance of acute thoracolumbar intervertebral disc herniations in dogs. Vet. Radiol. Ultrasound, v.41, p.396-402, 2000.

SMOLDERS, L.A.; BERGKNUT, N.; GRINWIS, G.C.M. et al. Intervertebral disc degeneration in the dog. Part 2: Chondrodystrophic and nonchondrodystrophic breeds. Vet. J.,v.195, p.292-299, 2013.

STIGEN, O. Calcification of intervertebral discs in the Dachshund: a radiographic study of $115 \mathrm{dogs}$ at 1 and 5 years. Acta Vet. Scand., v.37, p.229-237, 1996.

STIGEN, O.; KOLBJORNSEN, O. Calcification of intervertebral discs in the Dachshund: a radiographic and histopathologic study of 20 dogs. Acta Vet. Scand., v.49, p.1-7, 2007.

WIDMER, W.R.; THRALL, D.E. Canine and feline intervertebral disc disease, myelography, and spinal cord disease. In: THRALL, D.E. Textbook of veterinary diagnostic radiology. 4.ed. St. Louis: Elsevier Saunders, 2002. p.110-127. 